\title{
Multi-Objective Robust Scheduling Optimization Model of Wind, Photovoltaic Power, and BESS Based on the Pareto Principle
}

\author{
Guan Wang ${ }^{1}$, Zhongfu Tan ${ }^{1,2}$, Qingkun Tan ${ }^{1, * \mathbb{C}}$, Shenbo Yang ${ }^{1}$, Hongyu Lin ${ }^{1}$, Xionghua Ji ${ }^{2}$, \\ De Gejirifu ${ }^{1}$ and Xueying Song ${ }^{1}$ \\ 1 School of Economics and Management, North China Electric Power University, Beijing 102206, China; \\ wangguan@ncepu.edu.cn (G.W.); tzhf@ncepu.edu.cn (Z.T.); ysbo@ncepu.edu.cn (S.Y.);

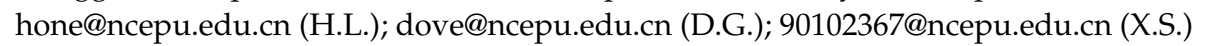 \\ 2 School of Economics and Management, Yan'an University, Yan'an 716000, China; ydglx_jxh@163.com \\ * Correspondence: tanqingkun@ncepu.edu.cn;
}

Received: 21 October 2018; Accepted: 31 December 2018; Published: 9 January 2019

\begin{abstract}
With the increasing proportion of distributed power supplies connected to the power grid, the application of a battery energy storage system (BESS) to a power system leads to new ideas of effectively solving the problem of distributed power grid connections. There is obvious uncertainty involved in distributed power output, and these uncertainties must be considered when optimizing the scheduling of virtual power plants. In this context, scene simulation technology was used to manage the uncertainty of wind power and photovoltaic output, forming a classic scenario. In this study, to reduce the influence of the uncertainty of wind and photovoltaic power output on the stable operation of the system, the time-of-use (TOU) prices and BESS were incorporated into the optimal scheduling problem that is inherent in wind and photovoltaic power. First, this study used the golden section method to simulate the wind and photovoltaic power output; second, the day-ahead wind and photovoltaic power output were used as the random variables; third, a wind and photovoltaic power BESS robust scheduling model that considers the TOU price was constructed. Finally, this paper presents the Institute of Electrical and Electronics Engineers (IEEE) 30 bus system in an example simulation, where the solution set is based on the Pareto principle, and the global optimal solution can be obtained by the robust optimization model. The results show that the cooperation between the TOU price and BESS can counteract wind and photovoltaic power uncertainties, improve system efficiency, and reduce the coal consumption of the system. The example analysis proves that the proposed model is practical and effective. By accounting for the influence of uncertainty of the optimal scheduling model, the actual operating cost can be reduced, and the robustness of the optimization strategy can be improved.
\end{abstract}

Keywords: TOU price; BESS; wind and photovoltaic power; the golden section method; robust scheduling optimization

\section{Introduction}

The randomness and fluctuation of wind and photovoltaic power can interrupt the system's grid connection. In 2016, China's total installed capacity of wind and photovoltaic power was $182.15 \mathrm{GW}$, ranked first in the world, but the average utilization of wind power generation was only $1728 \mathrm{~h}$. This is especially true of the "three North areas", wherein wind power resources are relatively rich: In this region, the average utilization of wind and photovoltaic power generation is only about $1445 \mathrm{~h}$. In order to realize large-scale wind power connected to the grid, it is of great practical significance to study the 
battery energy storage system (BESS) to control the output of wind and photovoltaic power, with the aim of optimizing the scheduling of a power system that uses wind and photovoltaic power [1,2].

The existing research on renewable energy primarily involves three aspects: Renewable energy power output forecasting, scheduling models, and solving algorithms. For renewable energy power output forecasting, there are mainly classical prediction methods, modern forecasting methods, and intelligent prediction methods [3]. In particular, the development of intelligent prediction algorithms greatly stimulates the progress of renewable energy power prediction technology. However, in practical applications, the prediction accuracy of these methods cannot yet meet the needs of system scheduling control. Many scholars have carried out specific research on the scheduling model and strategy of distributed energy uncertainty. According to cooperative game theory, in references $[4,5]$, the authors studied the joint system scheduling model with wind power and distribution companies. Cascaded hydro generators with variable wind power generation can improve System Energy Utilization Efficiency. In references [6,7], the authors studied the use of controllable loads to suppress the uncertainty of wind power output to improve revenue. Load management is an important aspect in the demand side response. It can improve the user's electricity habits by regulating the demand side load and achieve the target management of the target load management, which reduces the maximum load of the power grid, reduces the installed capacity of the system, and reduces the operating cost. Three types, namely peak clipping, valley filling, and peak shifting. In references [8,9], based on the pre-load forecast and wind power output forecast, the optimization method of wind power-pumped storage combined with daily operation is studied, and a new mode of grid output for wind power is proposed. Taking the benefit of wind power-pumped storage combined operation as the goal, a mixed integer programming model was established and solved by commercial optimization software. The test results show that the cooperation between the pumping power station and the wind farm can greatly reduce the negative impact of the randomness of wind power output on the grid operation, and the social benefits are significant. In reference [10], the authors studied a virtual power plant (VPP) economic scheduling model based on chance-constrained programming, which they used to manage multiple random variables, such as distributed power output and load forecasting error. In reference [11], the authors used a VPP as the energy management method of distributed energy and studied the optimal configuration model of a BESS for a large-scale distributed photovoltaic system. At present, scholars have performed little research on the optimal scheduling problem of BESSs and wind farms. In reference [12], the authors studied a VPP bidding strategy for electric vehicles and established a VPP joint bidding robust optimization model in the context of the energy market and regulation market. In references $[13,14]$, the authors studied multi-objective optimization scheduling of a system with participating electric vehicle. The model considers the impact of wind power operation on the environment. In references $[15,16]$, the authors proposed an interactive optimization scheduling model for power systems considering virtual power plants. In references [17,18], the authors used an energy management system to dispatch a cogeneration unit and a dispersed small hydropower unit that were aggregated by a VPP to realize the coordinated operation and reliable grid connection of a large number of Distributed Generations (DGs).

In reference [19], the stochastic programming method is used to study the wind power and pumped storage complementary operation scheduling strategies under island operation. However, most of the above literatures are optimized for the complementary scheduling of microgrids. The research on complementary optimized scheduling of large-scale renewable energy grid-connected is still in its infancy. In reference [20], the minimum abandonment rate, the minimum total operating cost, and the maximum benefit of the pumped storage power station are optimized. The fuzzy decision-making method is used to solve the multi-objective optimization model to plan the pumped storage capacity.

At present, domestic and foreign scholars have carried out certain research on wind power dispatching models and strategies. According to the cooperative game theory, in references [21], the authors studied the joint scheduling model of wind and light water VPP alone and distribution 
company. In reference [22], studied the VPP economic dispatch model and improved the profit by using the controllable load to suppress the uncertainty of wind power output. In reference [23], the paper studied the VPP medium-term optimal scheduling model in the context of electricity market. The model considers the market electricity price and the uncertainty of distributed power output. In reference [24], the VPP economic scheduling model based on chance constrained programming is studied. The opportunity constrained programming is used to deal with multiple random variables such as distributed power output and load forecasting error.

The above research efforts have obtained some results on wind power optimal scheduling models and strategies, but there are few studies on scheduling problems using a BESS. The stochastic programming method and the alternate tuning method are often used to address the uncertain risk in the scheduling model. This method takes the accurate probability distribution of the uncertainty factor as the premise; the amount of calculation required is large. On the other hand, the robust optimization method is applied less frequently. The influence of robust coefficient system optimization scheduling and the approach to determining the robustness coefficient under a given risk preference level needs further study. In the context of the above background, this paper presents the design and results of a VPP composed of a BESS, wind farm, and gas turbine, with the minimization of the operating cost and carbon emission as the objective function. Considering the uncertainty of a schedulable BESS and wind farm power output, a BESS and wind farm robust stochastic optimization scheduling model was constructed. The structure of the article is as follows.

First, a combined optimal scheduling model for wind and photovoltaic power and a BESS is proposed. The golden section method was applied to wind and photovoltaic power generation scenarios. Then, based on the robust optimization theory, the predicted results of the wind and photovoltaic power output were taken as random variables. In order to solve the proposed model, the Nash equilibrium criterion was used to obtain the optimal solution set and its front edge, and then the Mixed integer linear programming (MILP) was used to solve the robust optimization problem. There were four kinds of simulation scenarios set up, and the IEEE 30 bus system was used in the simulation analysis. Finally, an example is given to prove that the proposed robust stochastic optimization scheduling model and the optimization scheduling results are reliable and economical. The impact of the BESS and time-sharing electricity price on the operating cost and environment of the system was analyzed, and the results are presented.

\section{System Output Model}

\subsection{Wind and Photovoltaic Power Output Model}

The randomness and uncertainty of wind power and photovoltaic output depend on the randomness of the wind speed and light radiation in the region, and the wind speeds and light radiation is susceptible to weather factors and terrain conditions. Although wind power and photovoltaic output have randomness and uncertainty, a large quantity of statistical data indicates that wind speed is subject to the Weibull distribution; the specific formula of wind power output can be found in the literature [25]. The output curve of a photovoltaic power system generally satisfies the Beta distribution; the specific formula for photovoltaic power output can be found in the literature [26].

\subsubsection{Wind Power Output Model}

Wind turbine power is subject to the wind speed, but when the wind speed is lower than the cut into speed or higher than the cut-out speed, wind turbines do not generate electricity, the relationship between wind turbines power and wind speed is as follows: 


$$
g_{j, w}(t)= \begin{cases}0 \quad, v(t) \leq v_{\mathrm{i}, w} \text { or } v(t)>v_{\mathrm{o}, w} \\ g_{\mathrm{r}}\left(v(t)-v_{\mathrm{i}, w}\right)\left(v_{\mathrm{r}, w}-v_{\mathrm{i}, w}\right) \\ g_{\mathrm{r}}, & , v_{\mathrm{i}, w} \leq v(t) \leq v_{\mathrm{r}, w} \leq v(t) \leq v_{\mathrm{o}, w}\end{cases}
$$

Wherein: $g_{j, w}(t)$ is output of $\mathbf{j}$ wind turbine at time $\mathbf{t} ; g_{r}$ is the rated power of wind turbine; $v_{\mathrm{i}, w}$ and $v_{\mathrm{o}, w}$ are the cut into speed and the cut-out speed of wind turbine; $v_{r, w}$ is the rated speed of wind turbine; $v(t)$ is the speed of wind turbine at time $t$.

\subsubsection{Photovoltaic Power Model}

The output curve of photovoltaic system generally satisfies the Beta distribution, the specific formula is as follows:

$$
f(\theta)= \begin{cases}\frac{\Gamma(\alpha) \Gamma(\beta)}{\Gamma(\alpha)+\Gamma(\beta)} \theta^{\alpha-1}(1-\theta)^{\beta-1} & , 0 \leq \theta \leq 1, \alpha \geq 0, \beta \geq 0 \\ 0 & , \text { else }\end{cases}
$$

Wherein: $\alpha$ and $\beta$ is the parameter of the Beta distribution; $\theta$ is the radiosity correlation coefficient; the parameters of Beta are calculated by Formulas (3) and (4) as follows:

$$
\begin{gathered}
\beta=(1-\mu) \times\left(\frac{\mu \times(1+\mu)}{\delta^{2}}-1\right) \\
\alpha=\frac{\mu \times \beta}{1-\mu}
\end{gathered}
$$

Wherein: $\mu$ and $\sigma$ is mean and normal distribution of solar radiation; the probability of solar radiation can be calculated by Formula (5):

$$
P(\theta)=\int_{\theta_{c}}^{\theta_{d}} f(\theta) d \theta
$$

Wherein: $\theta_{c}$ and $\theta_{d}$ are upper and lower limits of solar radiation $\theta$; the formula for calculating the conversion of solar radiation into electrical energy is as follows:

$$
g_{m, p v}(t)=\varepsilon \times P(\theta) \times S_{P V} \times \theta_{t}
$$

Wherein: $P(\theta)$ is radiation intensity; $S_{p v}$ is area of photoelectric; $\theta_{t}$ is photoelectric conversion efficiency of photovoltaic array; $\varepsilon$ is output efficiency of photoelectric inverter.

\subsection{BESS Output Model}

A battery energy storage system (BESS) is charged at night and discharged in the daytime, which can improve the stability of the system and facilitate a wind and photovoltaic (PV) power connected grid.

$$
g_{k, s}^{\min }(t) \leq g_{k, s}(t) \leq g_{k, s}^{\max }(t)
$$

wherein $g_{k, s}^{\max }(t)$ and $g_{k, s}^{\min }(t)$ are the upper and lower limits of capacity for the BESS; $\mathrm{k}$ is number of the battery energy storage system; $s$ is battery energy storage system; $g_{k, s}(t)$ is the BESS capacity at time $t$. When the BESS is in the discharging state:

$$
g_{k, s}(t+1)=g_{k, s}(t)-\sum_{s=1}^{S} g_{k, s}^{d}(t)\left(1+\rho_{k, s}^{d}\right)
$$

When the BESS is in the charging state: 


$$
g_{k, s}(t+1)=g_{k, s}(t)+\sum_{k=1}^{N_{s}} g_{k, s}^{c}(t)\left(1-\rho_{k, s}^{c}\right)
$$

wherein $g_{k, s}^{d}(t)$ and $g_{k, s}^{c}(t)$ are the charging and discharging power of BESS $\mathrm{k}$ at time $\mathrm{t} ; g_{k, s}(t+1)$ is the power capacity of BESS $\mathrm{k}$ at time $\mathrm{t}+1$; $\mathrm{d}$ indicates that the BESS is discharged; $\mathrm{C}$ indicates that the BESS is charged; $\rho$ is loss of the BESS; $\rho_{k, s}^{d}$ and $\rho_{k, s}^{c}$ are the discharging and charging loss of the BESS.

The mathematical model of a control strategy for the charging and discharging of a BESS during a normal period is as follows.

$$
\left\{\begin{array}{lr}
0 \leq g_{k, s}(t) \leq g_{k, s}(t-1)-g_{k, s}(t)+\lambda_{\max } & \left|g_{k, s}(t-1)-g_{k, s}(t)\right|<\lambda_{\max \text { discharging }} \\
g_{k, s}(t-1)-g_{k, s}(t)+\lambda_{\max }>g_{k, s}(t) \geq g_{k, s}(t-1)-g_{k, s}(t)-\lambda_{\max } g_{k, s}(t)-g_{k, s}(t-1) \geq \lambda_{\max } \text { charging } \\
g_{k, s}(t-1)-g_{k, s}(t)+\lambda_{\max }<g_{k, s}(t) \leq g_{k, s}(t-1)-g_{k, s}(t)+\lambda_{\max } g_{k, s}(t)-g_{k, s}(t-1)<-\lambda_{\max } \text { discharging }
\end{array}\right.
$$

The mathematical model of a control strategy for the charging and discharging of a BESS during a valley period is as follows.

$$
\left\{\begin{array}{l}
g_{k, s}(t)-g_{k, s}(t-1)+\lambda_{\max }>g_{k, s}(t) \geq g_{k, s}(t)-g_{k, s}(t-1) g_{k, s}(t)-g_{k, s}(t-1) \geq 0 \text { charging } \\
g_{k, s}(t) \leq g_{k, s}(t)-g_{k, s}(t-1) \\
g_{k, s}(t-1)-\lambda_{k, s}(t)<g_{k, s}(t) \leq g_{k, s}(t-1)-g_{k, s}(t)-\lambda_{\max } g_{k, s}(t)-g_{k, s}(t)-g_{k, s}(t-1)<0 \text { discharging } \\
g_{k, s}(t-1)<-\lambda_{\max \text { discharging }}
\end{array}\right.
$$

The mathematical model of a control strategy for the charging and discharging of a BESS during a peak period is as follows.

$$
\left\{\begin{array}{l}
g_{k, s}(t)-g_{k, s}(t-1)+\lambda_{\max }>g_{k, s}(t) \geq g_{k, s}(t)-g_{k, s}(t-1) g_{k, s}(t)-g_{k, s}(t-1) \leq 0 \text { dicharging } \\
0 \leq g_{k, s}(t) \leq g_{k, s}(t)-g_{k, s}(t-1) \\
g_{k, s}(t)-g_{k, s}(t-1)-\lambda_{\max }<g_{k, s}(t)-g_{k, s}(t-1)>0 \text { charging } \\
g_{k, s}(t) \leq g_{k, s}(t)-g_{k, s}(t-1) g_{k, s}(t)-g_{k, s}(t-1) \geq \lambda_{\max } \text { charging }
\end{array}\right.
$$

wherein $\lambda_{\max }$ is the maximum amplitude of the output of wind and PV power connected to the system.

\section{System Robust Scheduling Model}

\subsection{Objective Function}

\subsubsection{System Operation Cost}

The minimum cost of system operation is taken as an objective function, and the specific objective function is as follows:

$$
E^{c}=\sum_{t=1}^{T} \sum_{i=1}^{I} \sum_{h=1}^{H}\left(C_{i, t}+C_{i, t}^{s S}+C_{t}^{s t o r}+C_{t}^{D R}\right)^{h}
$$

wherein $E^{c}$ is the cost of the scheduling system; $C_{i, t}$ and $C_{i, t}^{s S}$ are the fuel cost and start-stop cost for thermal power generation; $C_{t}^{s t o r}$ is the connected grid cost of the BESS.

$$
\begin{gathered}
C_{i, t}=\alpha_{i}+\beta_{i} g_{i t}+\gamma_{i}\left(g_{i t}\right)^{2} \\
C_{i, t}^{\text {ss }}=\left[u_{i t}\left(1-u_{i, t-1}\right)\right] N_{i t} \\
N_{i t}=\left\{\begin{array}{l}
N_{i}^{\text {hot }}, T_{i}^{\text {min }}<T_{i t}^{\text {off }} \leq H_{i}^{\text {off }} \\
N_{i}^{\text {cold }}, T_{i t}^{\text {off }}>H_{i}^{\text {off }}
\end{array}\right. \\
H_{i}^{\text {off }}=T_{i}^{\text {min }}+T_{i}^{\text {cold }}
\end{gathered}
$$

wherein $\alpha_{i}, \beta_{i}, \gamma_{i}$ are fuel cost coefficients of generator $\mathrm{i} ; N_{i}^{\text {cold }}$ is the cold start cost coefficient of generator i; $N_{i}^{\text {hot }}$ is the start-up cost of generator $\mathrm{i}$; $T_{i}^{\mathrm{min}}$ is the minimum allowable downtime of generator $i$; $T_{i t}^{\text {off }}$ is the continuous shutdown time of generator $i$; $T_{i}^{\text {cold }}$ is the cold start time of generator $\mathrm{i} ; H_{i}^{\text {off }}$ is the downtime and cold start time of generator i [27]. 
A BESS consuming power when it charges and discharges. The specific relationship is as follows:

$$
C_{s t o r}^{h}(t)=\sum_{s=1}^{N_{s}} B_{s}(t)\left[\left(g_{s}^{d}(t)-g_{s}^{c}(t)\right)\right]^{h}
$$

wherein $g_{s}^{d}(t)$ and $g_{s}^{c}(t)$ are the charging and discharging power of BESS s at time $t ; B_{s t o r}$ is the price of the BESS at time $t$.

\subsubsection{System Carbon Emissions}

Minimizing the carbon emissions of the system are taken as an objective function, and the specific relationship is as follows:

$$
E^{E}=a_{i, \mathrm{co}_{2}}\left(\mathrm{~g}_{i}\right)^{2}+b_{i, \mathrm{CO}_{2}} g_{i}+c_{i, \mathrm{co}_{2}}
$$

wherein $E^{E}$ is the total carbon emissions of the system; $a_{i, \mathrm{Co}_{2}}, b_{i, \mathrm{CO}_{2}}$, and $c_{i, \mathrm{CO}_{2}}$ are the carbon emission coefficients of generator i.

\subsection{Constraint Conditions}

The constraints of the model presented in this paper are as follows:

(1) Load balance

$$
\sum_{i=1}^{N_{G}} g_{i}(t) u_{i}(t)\left(1-\psi_{i}\right)+\sum_{s=1}^{S} g_{s}^{d}(t)\left(1-\rho_{s}^{d}\right)+\sum_{w=1}^{W} g_{w, p v}(t)\left(1-\varphi_{w}\right)=L^{0}(t)-\Delta L(t)+\sum_{s=1}^{S} g_{s}^{c}(t)\left(1+\rho_{s}^{c}\right)
$$

wherein $N_{G}$ is the number of generators of the system; $S$ is the number of BESSs; $W$ is the number of wind and photovoltaic power systems; $\psi_{i}$ is the generator power consumption rate of the system; $L^{0}(t)$ is the load of system; $\Delta L(t)$ is the transferred load under TOU; $\varphi_{w}$ is the wind and photovoltaic power consumption rate of the system.

(2) Power constraint of transmission line

The tie-line power constraint on the buses of the system can be expressed as follows:

$$
g_{\text {line }}^{\min } \leq g_{n}(t) \leq g_{\text {line }}^{\max }
$$

wherein $g_{n}(t)$ is the power of transmission line $\mathrm{n}$ at time $\mathrm{t} ; g_{\text {line }}^{\min }$ and $g_{\text {line }}{ }^{\max }$ are the maximum and minimum power of transmission line $\mathrm{n}$ at time $\mathrm{t}$.

Unit constraints mainly include upper and lower power constraints, climbing constraints, minimum start time constraints, and minimum downtime constraints, and are described in the literature [8].

\subsection{Uncertainty Set}

$g_{w, k}^{d}$ and $g_{w, k}^{u}$ are the minimum and maximum output range of wind power at time $\mathbf{t} ; g_{p v, m}^{d}$ and $g_{p v, m}^{u}$ are the minimum and maximum output range of photovoltaic power at time $t$. Their formulas are as follows:

$$
\begin{gathered}
g_{w, k}^{d} \leq g_{w, k} \leq g_{w, k}^{u} \\
g_{p v, m}^{d} \leq g_{p v, m} \leq g_{p v, m}^{u}
\end{gathered}
$$

The absolute predictive error of wind and photovoltaic power is constrained by the 1-norm, and the parameter $\Gamma$ is the uncertainty coefficient.

$$
\sum_{k=1}^{k}\left|g_{w t, k}^{a}-g_{w t, k}^{f}\right| / g_{w t, k}^{f} \leq \Gamma_{1}
$$




$$
\sum_{k=1}^{k}\left|g_{p v, m}^{a}-g_{p v, m}^{f}\right| / g_{p v, m}^{f} \leq \Gamma_{2}
$$

wherein $\Gamma_{1}$ and $\Gamma_{2}$ are the uncertainty coefficients of wind and photovoltaic power output. The above problem is a linear constrained two-objective optimization.

\subsection{Solving Algorithm}

The objective function is a second-order function, and the constraint is a linear function. This model is a bi-objective quadratic convex programming problem. The algorithm flow is shown in Figure 1.

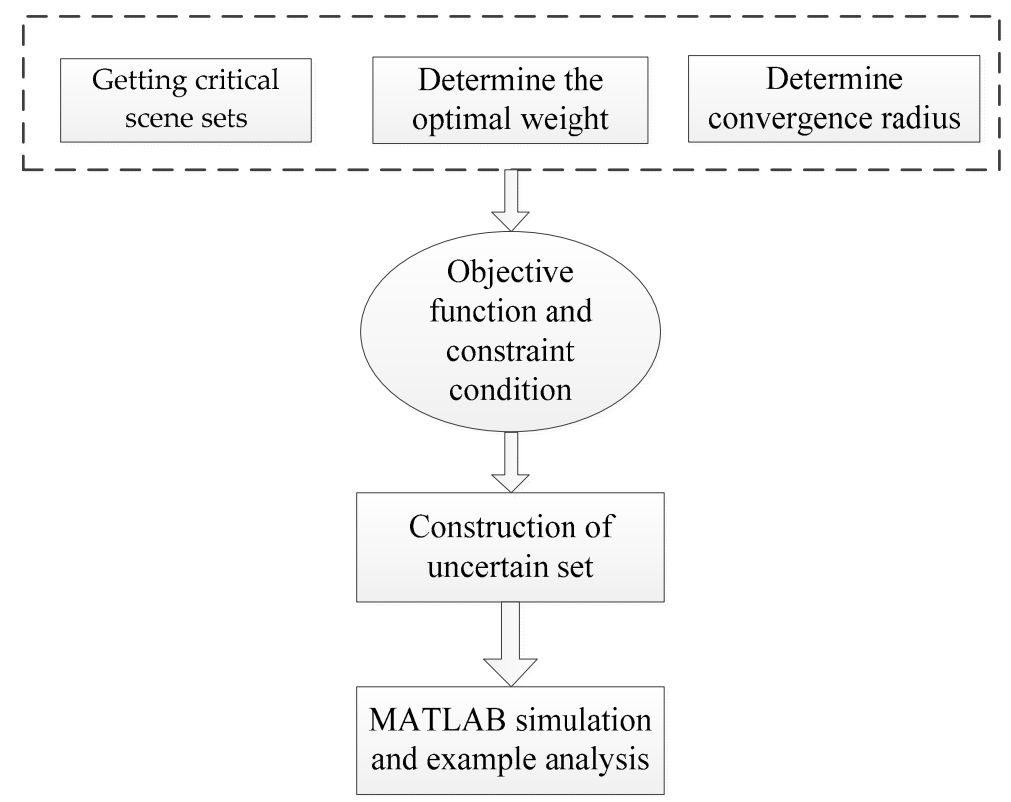

Figure 1. The algorithm flowchart.

The Pareto optimal set is $X^{P}$, the Pareto front is $P^{R}$, and the defining formula is as follows:

$$
X^{P}=\cup_{\lambda \in(0,1)} x(\lambda), P^{R}=\cup_{\lambda \in(0,1)} P_{E C} x(\lambda)
$$

wherein $\lambda \in(0,1), x(\lambda)$ is the optimal solution to two optimization problems, with $\lambda$ as the parameter in Formula (27):

$$
\min \left\{\lambda E^{c}+(1-\lambda) E^{E}\right\}
$$

wherein $P_{E C} x(\lambda)$ is a point of a two-dimensional coordinate plane; $\lambda$ is the weight of the objective function; $E^{c}(x(\lambda))$ is the abscissa, and $E^{E}(x(\lambda))$ is the ordinate. Using the Nash equilibrium principle, a scheduling strategy that considers both generation cost and carbon emissions can be found in the Pareto optimal set $X^{P}$. The negotiation solution to the optimization problem is obtained by Formula (28):

$$
\max \left\{\left(E_{D}^{C}-E^{C}(x)\right)\left(E_{D}^{E}-E^{E}(x)\right)\right\}
$$

wherein $x \in X^{P},\left\{E_{D}^{C}, E_{D}^{E}\right\}$ are the breaking points of negotiation and the worst result that can be accepted as the optimal solution to two objective functions.

$$
\begin{aligned}
& E_{D}^{C}=E^{C}\left(x^{E}\right), x^{E}=\operatorname{argmin}\left\{E^{E}\right\} \\
& E_{D}^{E}=E^{E}\left(x^{C}\right), x^{C}=\operatorname{argmin}\left\{E^{C}\right\}
\end{aligned}
$$


The Pareto set can be parameterized by the single variable $\lambda$, and the golden section method can be used to search for the negotiation solution to the set $X^{P}$. The golden section method reduces the interval containing the extremum until the search area is less than the given error. $C^{S}$ is the key field set.

Algorithm 1:

Step 1: Get the critical scene sets $C^{S}$, assume $\lambda^{a}=0, \lambda^{b}=1$, and apply the Nash negotiation principle, which can calculate the negotiation breakdown points $E_{D}^{C}, E_{D}^{E}$. The transfer step for setting the golden section point is $\phi=0.618, \varepsilon>0$.

Step 2: $\lambda^{1}=\phi \lambda^{a}+(1-\phi) \lambda^{b}, \lambda^{2}=(1-\phi) \lambda^{a}+\phi \lambda^{b}$. At the points $\lambda=\lambda^{1}$ and $\lambda=\lambda^{2}$, to get the optimal solution of objective function $x^{1}, x^{2}$, use Formulas (31) and (32) to calculate $f^{1}, f^{2}$; the defining formulas are as follows:

$$
\begin{aligned}
& f^{1}=-\left(E_{D}^{C}-E^{C}\left(x^{1}\right)\right)\left(E_{D}^{E}-E^{E}\left(x^{1}\right)\right) \\
& f^{2}=-\left(E_{D}^{C}-E^{C}\left(x^{2}\right)\right)\left(E_{D}^{E}-E^{E}\left(x^{2}\right)\right)
\end{aligned}
$$

Step 3: If $\lambda^{b}-\lambda^{a}<\varepsilon$, execute Step 4 .

Step 4: If $f_{1}<f_{2}$, order $\lambda^{b}=\lambda^{2}, \lambda^{2}=\lambda^{1}, f^{2}=f^{1}, \lambda^{1}=\phi \lambda^{a}+(1-\phi) \lambda^{b} ; \lambda^{1}$ is into objective function Formula (30). The optimal solution $x^{1}$ is substituted for Formula (28), which can get $f^{1}$. If $f^{1}$ is obtained, execute Step 3; otherwise, order $\lambda^{a}=\lambda^{1}, \lambda_{1}=\lambda_{2}, f^{1}=f^{2}, \lambda^{2}=(1-\phi) \lambda^{a}+\phi \lambda^{b} ; \lambda^{2}$ is substituted into objective function Formula (31). The optimal solution $x^{2}$ is substituted for Formula (35), which can get $f^{2}$. Then, execute Step 3.

Step 5: End: the optimal scheduling policy $x$ is obtained, and $\lambda^{*}=0.5\left(\lambda^{1}+\lambda^{2}\right)$.

In Algorithm 1, the interval $\left[\lambda^{a}, \lambda^{b}\right]$ is decreased to $61.8 \%$ of the original length of each iteration, and the algorithm will terminate after $\mathrm{N}$ iterations. In the algorithm, $a$ is the maximum integer and is not greater than $[a]$. Algorithm 1 provides the optimal weight, which satisfies the Nash negotiation criterion, and the negotiation principle provides a reasonable result from the choice of compromise solution.

Algorithm 2:

Step 1: Get the critical scene sets $C^{S}=\varnothing$.

Step 2: Use $C^{S}$ and algorithm 1 to solve the main problem of relaxation:

$$
\min \left\{E^{C}, E^{E}\right\}
$$

Step 3: Fix the current $x$ value to solve MILP. The parameter $w$ is part of the optimal solution to $w^{s}$, and the optimal value is $R$. If $R=0$, terminate the solution process and return the pre-schedule value $x$; otherwise, update $C^{S}=C^{S} \cup w^{S}$ and the constraint conditions, then return to Sep 2.

Step 4: When wind and photovoltaic power output is observed, the following clean scheduling problem is solved, and the actual scheduling strategy is obtained:

$$
\begin{gathered}
E^{c}=\sum_{t=1}^{T} \sum_{i=1}^{I} \sum_{h=1}^{H} p_{h}\left(C_{i, t}+C_{i, t}^{s s}+C_{t}^{s t o r}+C_{t}^{D R}\right)^{h} \\
E^{E}=a_{i, \mathrm{co}_{2}}\left(\mathrm{~g}_{i}\right)^{2}+b_{i, \mathrm{co}_{2}} g_{i}+c_{i, \mathrm{co}_{2}}
\end{gathered}
$$

wherein $E^{c}$ is the cost of the scheduling system; $E^{E}$ is the total carbon emissions of the system. 


\section{Simulation Studies}

\subsection{Simulation Scenario Setting}

This paper establishes four system simulation scenarios:

Case 1: Basic scenario 1, which does not include the BESS and TOU price.

Case 2: The BESS is included in the system, but the TOU price is not included.

Case 3: The BESS is not included in scenario 3, but the TOU price is included. The load transfer of the peak time is no more than $25 \%$ of the original load.

Case 4: Scenario 4 includes the TOU price and the BESS in the system.

\subsection{Basic Data}

In this work, the IEEE 30 bus 10-generator system was used as the simulation system, with the following values: The rated capacity of two wind farms is $200 \mathrm{MW}$, the rated capacity of the photovoltaic system is $50 \mathrm{MW}$, the rated capacity of the BESS is $50 \mathrm{MW}$, and the operation parameters of the generators are as listed in Table 1.

Table 1. Operation parameters of generators.

\begin{tabular}{ccccccccccccc}
\hline & $\boldsymbol{a}_{\boldsymbol{i}}$ & $\boldsymbol{b}_{\boldsymbol{i}}$ & $\boldsymbol{c}_{\boldsymbol{i}}$ & Climbing Rate & $\boldsymbol{d}_{\boldsymbol{i}}$ & $\boldsymbol{e}_{\boldsymbol{i}}$ & $f_{\boldsymbol{i}}$ & $\boldsymbol{g}_{\min }$ & $\boldsymbol{g}_{\max }$ & $\boldsymbol{a}_{\boldsymbol{i}}^{\mathbf{c o}_{2}}$ & $\boldsymbol{b}_{\boldsymbol{i}}^{\mathbf{c o}_{2}}$ & $\boldsymbol{c}_{\boldsymbol{i}}^{\mathbf{c o}_{2}}$ \\
\hline$\# 1$ & 100 & 18 & 0.04 & 6.4 & 0.4276 & 1 & 0.0032 & 100 & 600 & $3.02 \times 10^{-5}$ & 0.822 & 22.8 \\
$\# 2$ & 200 & 10 & 0.05 & 8.6 & 0.03579 & 1 & 0.0034 & 100 & 650 & $2.95 \times 10^{-5}$ & 0.824 & 23.5 \\
$\# 3$ & 200 & 17 & 0.04 & 9.8 & 0.0248 & 1 & 0.0021 & 250 & 800 & $3.21 \times 10^{-5}$ & 0.830 & 24.1 \\
$\# 4$ & 100 & 15 & 0.1 & 4.2 & 0.01153 & 1 & 0.002 & 300 & 1000 & $4.65 \times 10^{-5}$ & 0.843 & 22.7 \\
$\# 5$ & 200 & 20 & 0.05 & 10.2 & 0.5276 & 1 & 0.0035 & 100 & 250 & $6.17 \times 10^{-5}$ & 0.861 & 19.3 \\
$\# 6$ & 220 & 22 & 0.05 & 10.8 & 0.6276 & 1 & 0.0038 & 100 & 150 & $8.79 \times 10^{-5}$ & 0.833 & 15.3 \\
\hline
\end{tabular}

The peak-valley periods are shown in Table 2. The electricity price in the normal period is 436 yuan/MW·h, and the peak period and valley period electricity prices are $25 \%$ higher and lower than that in the normal period.

Table 2. System time division.

\begin{tabular}{cccc}
\hline Period & Valley Period & Normal Period & Peak Period \\
\hline Time & $0: 00-5: 00 ; 21: 00-24: 00$ & $5: 00-8: 00 ; 14: 00-19: 00$ & $8: 00-14: 00 ; 19: 00-21: 00$ \\
\hline
\end{tabular}

In order to test the proposed model, the above optimization model is implemented by using MATLAB software and object-oriented programming technology. The analog power system used is a simple system based on the IEEE 30-node test system, and the power generation technology, capacity, load, and capacity of some lines of the original system are adjusted and assumed. The distribution of wind and photovoltaic power and the generators in the system are shown in Figure 2.

In this study, a robust scheduling model for wind power, photovoltaic power, and a BESS was constructed. For convenient analysis, the short-term prediction of wind and photovoltaic power output was not the focus of this study [27].

\subsection{Case Analysis}

According to the day-ahead scheduling robust scheduling optimization model, with an optimized structure of generator output, the large-capacity generator output increases. Figure 3 gives the Pareto frontier and the negotiation solution, and the weight of the negotiation solution is $\lambda=0.0511$. 


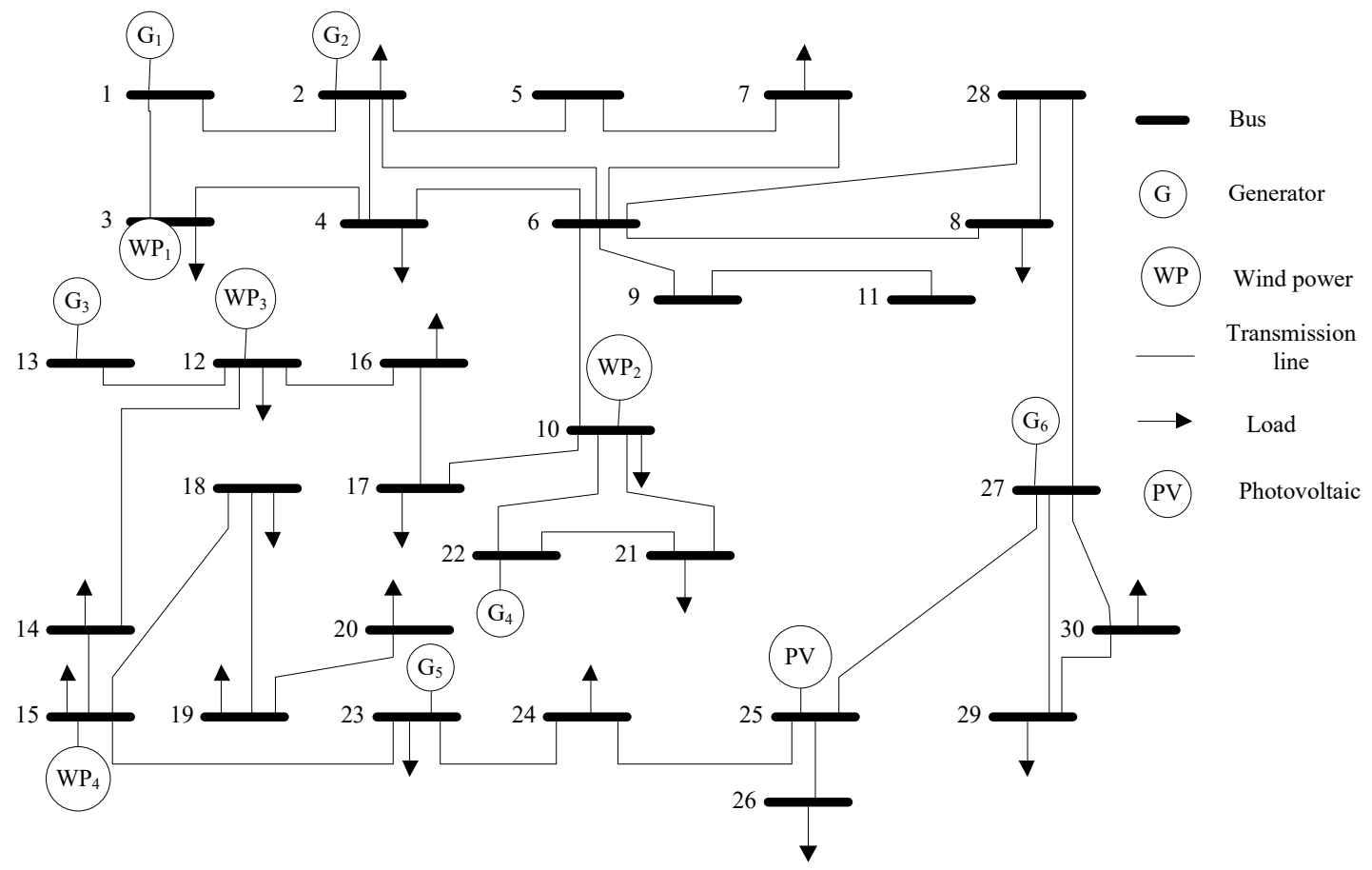

Figure 2. Topology diagram of the PJM30 bus system.

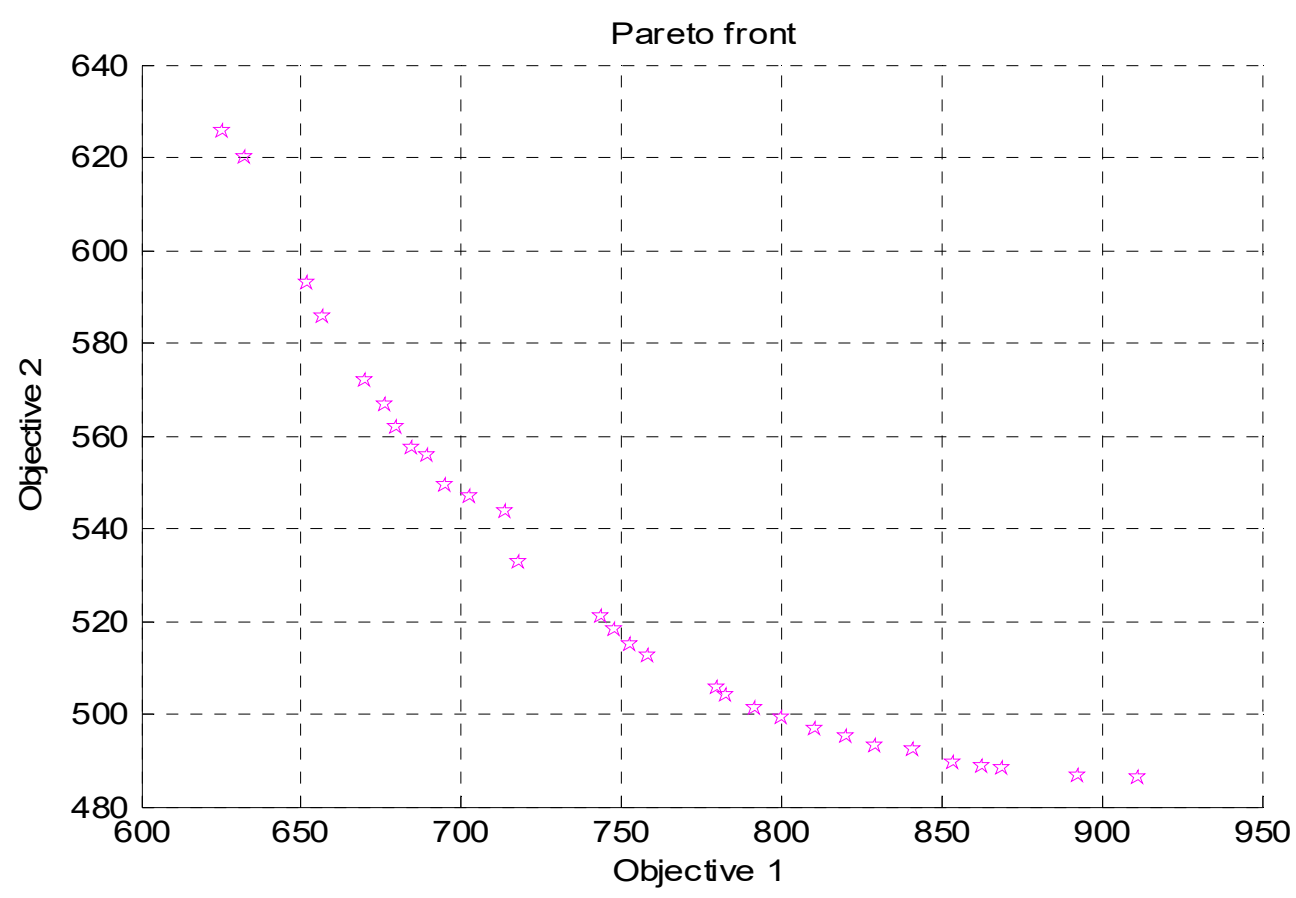

Figure 3. Pareto frontier and negotiation solution.

The robust scheduling optimization model can restrain the randomness of the wind and photovoltaic power output, increase the grid-connected power of the wind and photovoltaic power, and reduce the cost of system start-up and shutdown. Figure 4 is the day-ahead generator output structure. 


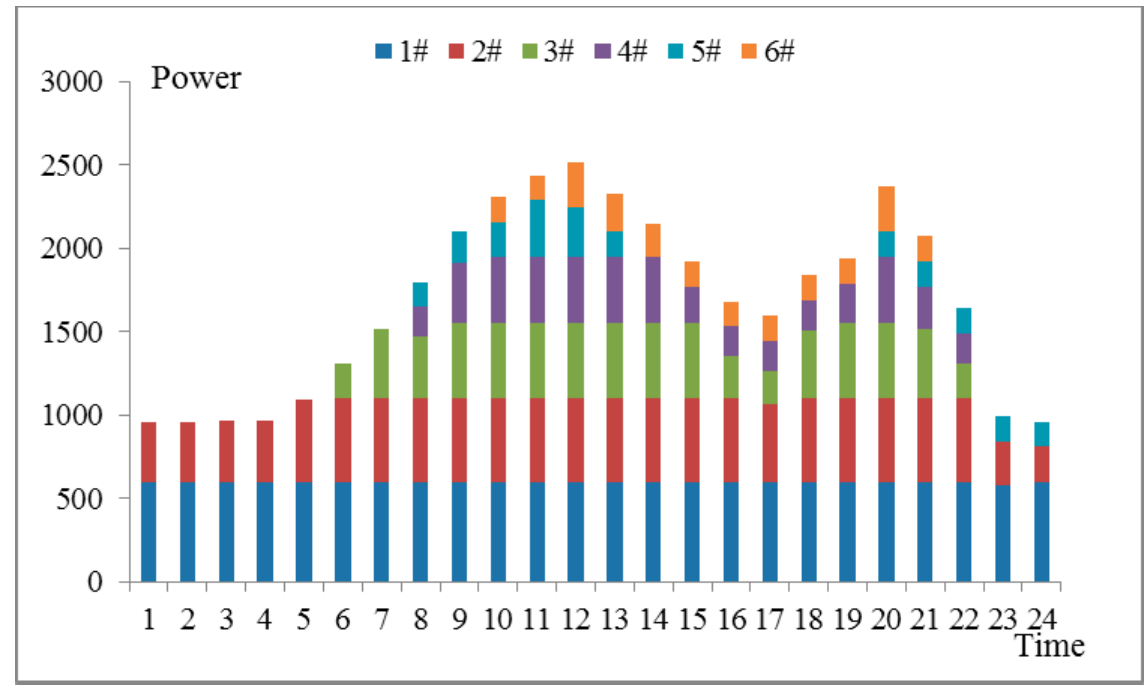

Figure 4. Generator output structure (MW).

\subsection{Comparison of Four Scenarios}

The inclusion of the TOU price and BESS can optimize the distribution of the load, reduce the peak period load, and increase the valley period load. Table 3 shows the operation optimization results of the four above-described scenarios.

Table 3. System operation optimization results for four scenarios.

\begin{tabular}{ccccccccc}
\hline & \multicolumn{3}{c}{ System Load Structure (\%) } & \multicolumn{2}{c}{ System Load } & $\begin{array}{c}\text { Wind and } \\
\text { Photovoltaic } \\
\text { Power }\end{array}$ & Thermal Power \\
\cline { 2 - 9 } Case & $\begin{array}{c}\text { Valley } \\
\text { Period }\end{array}$ & $\begin{array}{c}\text { Normal } \\
\text { Period }\end{array}$ & $\begin{array}{c}\text { Peak } \\
\text { Period }\end{array}$ & $\begin{array}{c}\text { Maximum } \\
\text { Load } \\
\text { (MW) }\end{array}$ & $\begin{array}{c}\text { Minimum } \\
\text { Load } \\
\text { (MW) }\end{array}$ & $\begin{array}{c}\text { Grid } \\
\text { Connected } \\
\text { Power (MWh) }\end{array}$ & $\begin{array}{c}\text { Grid } \\
\text { Connected } \\
\text { Power (MWh) }\end{array}$ & $\begin{array}{c}\text { Consumption } \\
\text { (g/kW) }\end{array}$ \\
\hline Case1 & 25.3 & 33.2 & 41.5 & 2700 & 900 & 8432 & 36,468 & 328.6 \\
\hline Case2 & 25.5 & 33.3 & 41.2 & 2620 & 950 & 8561 & 36,339 & 327.5 \\
\hline Case3 & 27.3 & 33.9 & 38.8 & 2350 & 900 & 8666 & 30,894 & 325.4 \\
\hline Case4 & 27.4 & 34 & 38.5 & 2220 & 980 & 8983 & 30,357 & 323.9 \\
\hline
\end{tabular}

Based on the data analysis in Table 4, the incorporation of the BESS and TOU price can effectively reduce the output of thermal power generation and the coal consumption of the system. In terms of energy savings and carbon emissions, the robust optimization model can reduce the total coal consumption of the system and the coal consumption rate of the system, and these results have obvious economic and environmental benefits. Further, the inclusion of the BESS and TOU price influences the system load, thermal power plant output structure, and wind and photovoltaic power output. Figure 5 is the system load curve for the four scenarios.

Table 4. Thermal power output structure in four scenarios (MW).

\begin{tabular}{ccccc}
\hline Generator & Case $\mathbf{1}$ & Case 2 & Case 3 & Case 4 \\
\hline $1 \#$ & 14,400 & 14,400 & 14,400 & 14,400 \\
$2 \#$ & 8571 & 8908 & 7526 & 7326 \\
$3 \#$ & 6449 & 6835 & 5217 & 5017 \\
$4 \#$ & 3312 & 3772 & 3032 & 2938 \\
$5 \#$ & 2800 & 2341 & 680 & 677 \\
$6 \#$ & 812 & 0 & 0 & 0 \\
\hline
\end{tabular}




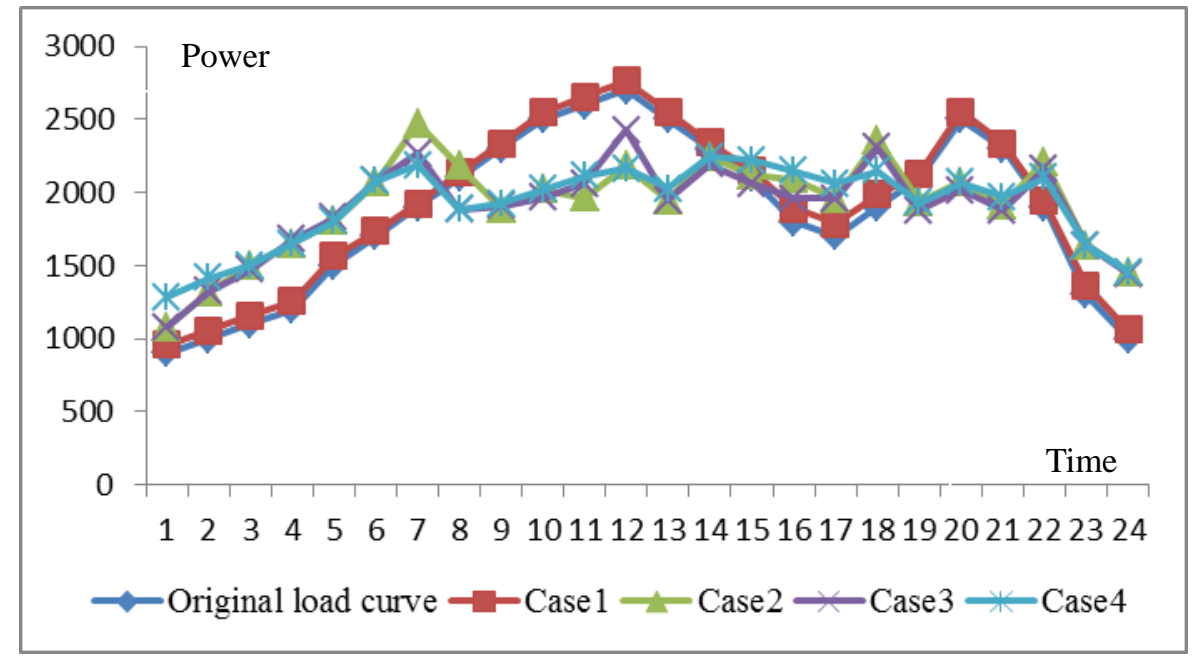

Figure 5. Load curves for four scenarios (MW).

In this part, the main task is to analyze the influence of application of the BESS and TOU price on the system load. The BESS can transfer the peak period load to the valley period, however it does not reduce the total load, which is shown by the results of scenario 1 and scenario 2; the TOU price can reduce the total load and the peak period load, which is shown by the result of scenario 1 .

Applying the BESS and TOU price has an influence on the generator structure: It can optimize the structure of the thermal power output, enhance the utilization efficiency of the generator, reduce the number of generators participating in reserve service output, reduce the number of thermal power start-ups, and reduce the coal consumption of the system. Table 4 is the thermal power output structure of the four scenarios. Comparing the four scenarios, the inclusion of the TOU price and the BESS can optimize the system operation.

In the fourth scenario, the simulation result shows that the BESS and TOU price can improve the system's wind and photovoltaic power connected grid output, and the wind power and photovoltaic capacity cannot be the same as the connected grid rate. Figure 6 is the wind and photovoltaic power connected grid capacity in the four scenarios.

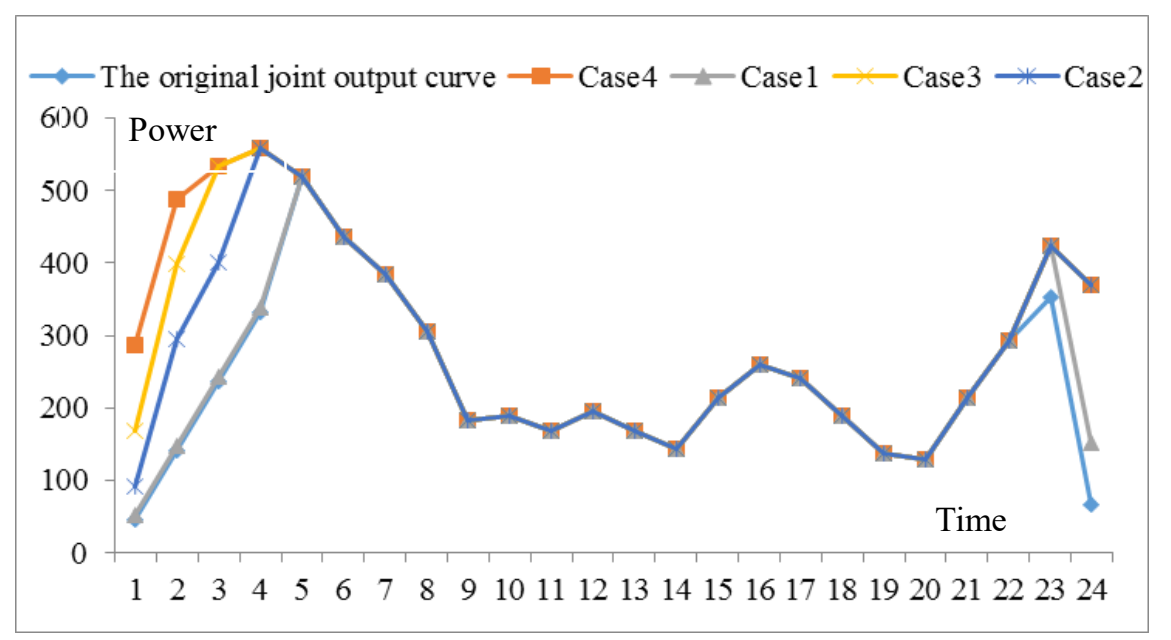

Figure 6. Wind and photovoltaic power connected grid capacity in four scenarios (MW).

The discharging and charging strategy of the BESS is shown in Table 5. In the second scenario, the BESS is charged during the valley period and normal period, and the total charged electricity is 284.2 $\mathrm{MW} \cdot \mathrm{h}$, which is discharged during the peak period. In the fourth scenario, the BESS is discharged 
and charged during every period. Contrasting scenario 2 and scenario 4 , in the fourth scenario, the connected grid capacity of the BESS is more than that in the other scenario. Thus, the BESS can result in more system operating cost savings when combined with the TOU price.

Table 5. BESS operating results.

\begin{tabular}{ccccccc}
\hline \multirow{2}{*}{ Case } & \multicolumn{2}{c}{ Valley Period } & \multicolumn{2}{c}{ Normal Period } & \multicolumn{2}{c}{ Peak Period } \\
\cline { 2 - 6 } & Charging & Discharging & Charging & Discharging & Charging & Discharging \\
\hline Case 2 & 160 & $\times$ & 124.2 & $\times$ & $\times$ & 270 \\
Case 4 & 198.6 & 18.3 & 157.2 & 40 & $\times$ & 261.2 \\
\hline
\end{tabular}

\section{Conclusions}

The randomness of wind and photovoltaic power output means that wind and photovoltaic power cannot be connected to the grid. The incorporation of the BESS and TOU price influences the system load and generator output structure. This paper presents a robust scheduling optimization model for the joint scheduling of wind and photovoltaic power. The model incorporated a BESS with the TOU price. The golden section algorithm and Nash equilibrium principle was applied to manage the value ranges of the objective function. The results of the examples show that:

(1) In order to account for the cost of power generation and carbon emissions, a compromise can be achieved by a reasonable application of the Nash equilibrium principle of negotiation with the golden section search algorithm compromise solution Pareto solution set. A decomposition algorithm for robust scheduling is thus proposed.

(2) A robust scheduling model based on modified day-ahead scheduling optimizes the generator output structure, reduces the net load that generators bear, reduces the cost of the power generation system, and increases the system capacity of the wind and photovoltaic power connected grid.

(3) From the simulation results, the wind and photovoltaic power connected grid capacity increases, using the BESS as a reserve. On the other hand, the generator sacrifices power generation profits. In order to garner enthusiasm for the inclusion of the BESS and generator in the coordinated operation of wind and photovoltaic power, it is necessary to design a reasonable benefit allocation mechanism, where some economic benefits will be used to subsidize the BESS and generators.

Author Contributions: Z.T. guided the research; G.W. and Q.T. established the model, implemented the simulation and wrote this article; S.Y. collected references; X.J., H.L., and X.S. revised the language of this article. It should be noted that the whole work was accomplished by the authors collaboratively. All authors read and approved the final manuscript.

Funding: Project Supported by National Natural Science Foundation of China (71573084) and Beijing Municipal Social Science Foundation (16JDYJB044).

Conflicts of Interest: The authors declare no conflict of interest. It should be noted that the whole work was accomplished by the authors collaboratively. All authors read and approved the final manuscript.

\section{References}

1. Bozchalui, M.C.; Hashmi, S.A.; Hassen, H.; Cañizares, C.A.; Bhattacharya, K. Optimal operation of residential energy hubs in smart grids. IEEE Trans. Smart Grid 2012, 3, 1755-1766. [CrossRef]

2. Zhang, X.; Shahidehpour, M.; Alabdulwahab, A. Optimal Expansion Planning of Energy Hub with Multiple Energy infrastructures. IEEE Trans. Smart Grid 2015, 6, 2302-2311. [CrossRef]

3. Lu, N.; Zhou, J.; He, Y. Particle swarm optimization-based neural network model for short-term load forecasting. Power Syst. Prot Control 2010, 38, 65-68.

4. Sedghi, M.; Ahmadian, A.; Aliakbar-Golkar, M. Optimal Storage Planning in Active Distribution Network Considering Uncertainty of Wind Power Distributed Generation. IEEE Trans. Power Syst. 2015, 31, 304-316. [CrossRef]

5. Abreu, L.V.L.; Khodayar, M.E.; Shahidehpour, M.; Wu, L. Risk-constrained coordination of cascaded hydro generators with variable wind power generation. IEEE Trans. Sustain. Energy 2012, 3, 359-368. [CrossRef] 
6. Xiang, X.; Song, Y.; Hu, Z.; Xu, Z. Research on optimal time of use price for electric vehicle participating V2G. Proc. CSEE 2013, 33, 15-26.

7. Chen, H.; Xuan, P.; Wang, Y.; Tan, K.; Jin, X. Key technologies for integration of multi-type renewable energy sources: Research on multi-time frame robust scheduling dispatch. IEEE Trans. Smart Grid 2015, 7, 471-480. [CrossRef]

8. Zou, Y.; Yang, L. Synergetic dispatch models of a wind/PV/hydro. Power Syst. Technol. 2015, 39, 1855-1860.

9. Chen, J.; Wu, W.; Zhang, B. A robust interval wind power dispatch method considering the tradeoff between security and Economy. Proc. CSEE 2014, 34, 1033-1041.

10. Coelho, V.N.; Coelho, I.M.; Coelho, B.N. Multi-objective BESS power dispatching using plug-in vehicles in a smart-micro grid. Renew. Energy 2016, 89, 730-742. [CrossRef]

11. Wanc, L.; Sinch, C. Stochastic combined heat and power dispatch based on multi-objective particle swarm optimization. Int. J. Electr. Power Energy Syst. 2008, 30, 226-234. [CrossRef]

12. Fan, S.; Ai, Q.; He, X. Risk analysis on dispatch of virtual power plant based on chance constrained programming. Proc. CSEE 2015, 39, 66-75.

13. Liu, Z.; Wen, F.; Ledwich, G. Optimal siting and sizing of distributed generators in distribution systems considering uncertainties. IEEE Trans. Power Deliv. 2011, 26, 2541-2551. [CrossRef]

14. Guandalini, G.; Campanari, S.; Romano, M.C. Power-to-gas plants and gas turbines for improved wind dispatchability: Energy and economic assessment. Appl. Energy 2015, 147, 117-130. [CrossRef]

15. Dueñas, P.; Leung, T.; Gil, M.; Reneses, J. Gas-electricity coordination in competitive markets under: Renewable uncertainty. IEEE Trans. Power Syst. 2015, 30, 123-131. [CrossRef]

16. Clegg, S.; Mancarella, P. Integrated modeling and assessment of the operational impact of power-to-gas(P2G) on electrical and gas transmission networks. IEEE Trans. Sustain. Energy 2016, 6, 1234-1244. [CrossRef]

17. Correa-Posada, C.M.; Sanchez-Martin, P. Security-constrained optimal power and natural-gas flow. IEEE Trans. Power Syst. 2014, 29, 1780-1787. [CrossRef]

18. Salimi, M.; Ghasemi, H.; Adelpour, M.; Vaez-ZAdeh, S. Optimal planning of energy hubs in interconnected energy systems: A case study for natural gas and electricity. IET Gener. Transm. Distrib. 2015, 9, 695-707. [CrossRef]

19. Ntomaris, A.V.; Bakirtzis, A.G. Stochastic scheduling ofhybrid power stations in insular power systems with high wind penetration. IEEE Trans. Power Syst. 2016, 31, 3424-3436. [CrossRef]

20. Hozouri, M.A.; Abbaspour, A.; Fotuhi-Firuzabad, M.; Moeini-Aghtaie, M. On the use of pumped storage for wind energy maximization in transmission-constrained power systems. IEEE Trans. Power Syst. 2015, 30, 1017-1025. [CrossRef]

21. Wenlue, D.; Qun, W.; Li, Y. A coordinated dispatching model for a distribution utility and virtual power plants with wind/photovoltaic/hydro generators. Autom. Electr. Power Syst. 2015, 39, 75-81.

22. Mohammadi, J.; Rahimi-kian, A. Aggregated wind power and flexible load offering strategy. IET Renew. Power Gener. 2007, 5, 439-447. [CrossRef]

23. Pandzic, H.; Kuzle, I. Virtual power plant mid-term dispatch optimization. Appl. Energy 2013, 101, $134-141$. [CrossRef]

24. Sun, Y.; Wu, J.; Li, G.; He, J. Dynamic economic dispatch considering wind power penetration based on wind speed forecasting and stochastic programming. Proc. CSEE 2009, 29, 41-47.

25. Wei, L.; Zhao, B.; Wu, H. Optimal allocation model of BESS system in virtual power plant environment with a high penetration of distributed photovoltaic generation. Autom. Electr. Power Syst. 2015, 39, 66-74.

26. Carrion, M.; Arroyo, J.M. A computationally efficient mixed-integer linear formulation for the thermal unit commitment problem. IEEE Trans. Power Syst. 2006, 21, 1371-1378. [CrossRef]

27. Zhang, W.; Wang, X.; Wu, X.; Yao, L. An analysis model of power system with large-scale wind power and transaction mode of direct power purchase by large consumers involved in system scheduling. Proc. CSEE 2015, 35, 2927-2995.

(C) 2019 by the authors. Licensee MDPI, Basel, Switzerland. This article is an open access article distributed under the terms and conditions of the Creative Commons Attribution (CC BY) license (http:/ / creativecommons.org/licenses/by/4.0/). 